\title{
Error Estimates for a Chebyshev Quadrature Method
}

\author{
By N. K. Basu
}

\begin{abstract}
Filippi [1] has proposed a quadrature scheme for any function $f(x)$ in $[-1,1]$, based on expanding the integrand in a series of Chebyshev polynomials of the second kind. In this paper the error associated with this quadrature method when applied to analytic functions has been investigated in detail.
\end{abstract}

Introduction. A Chebyshev polynomial of the second kind is $U_{n-1}(x) \equiv(1 / n) T_{n}^{\prime}(x)$ where $T_{n}(x)$ is the Chebyshev polynomial of the first kind of degree $n$, defined by $T_{n}(x)=\cos n \theta$ with $x=\cos \theta$. Accordingly, we shall follow Filippi and consider the expansion of function in terms of the $T_{n}^{\prime}(x)$ instead of $U_{n}(x)$.

Let $f(x)$, a function of bounded variation in $[-1,1]$, be expanded in a series of $T_{s}^{\prime}(x)$ as

$$
f(x)=\sum_{n=1}^{\infty} a_{n} T_{n}^{\prime}(x)
$$

where

$$
a_{n}=\frac{2}{\pi n^{2}} \int_{-1}^{1}\left(1-x^{2}\right)^{1 / 2} T_{n}^{\prime}(x) f(x) d x .
$$

In general, the integral in (2) cannot be evaluated explicitly and recourse has to be made to approximate methods for evaluating $a_{n}$ and then to obtain a suitable polynomial approximation to $f(x)$.

Filippi [1], has approximated the function $f(x)$ by a polynomial $\psi_{N-1}(x)$ of degree $N-1$, by collocation with $f(x)$ at the $N$-points, which are the zeros of $T_{N+1}^{\prime}(x)$ and has obtained a quadrature formula for $f(x)$ by integrating $\psi_{N-1}(x)$.

In the first section of this paper, the contour integral estimate of error $\psi_{N-1}(x)=$ $f(x)-\psi_{N-1}(x)$ is considered in brief and in the subsequent sections the error in the Filippi quadrature scheme for analytic functions is discussed. Analogous investigation on Clenshaw-Curtis quadrature [2] and Gaussian quadrature have been made by Chawla [3], [4] and Chawla and Jain [5].

1. Contour Integral Estimate of $\Psi_{N-1}(x)$. Following Filippi [1], let $f(x)$ be approximated by a polynomial $\psi_{N-1}(x)$ of degree $N-1$ over the zeros of $T_{N+1}^{\prime}(x)$, so that

$$
\psi_{N-1}(x)=\sum_{n=1}^{N} B_{n, N} T_{n}^{\prime}(x) \cdot \mathbf{J}
$$

Received January 14, 1969, revised January 30, 1970.

AMS 1969 subject classifications. Primary 6555, 6580.

Key words and phrases. Chebyshev quadrature method, function of bounded variation, expansion, Chebyshev polynomials of second kind, Lagrange interpolation polynomial, analytic functions, contour integral estimate of error. 
The coefficients $B_{n, N}$ are calculated by trigonometric interpolation as in [1].

$$
B_{n, N}=\frac{2}{n^{2}(N+1)} \sum_{i=1}^{N}\left(1-x_{i}^{2}\right) T_{n}^{\prime}\left(x_{i}\right) f\left(x_{i}\right),
$$

where

$$
x_{i}=\cos \frac{\pi i}{N+1}, \quad i=1(1) N .
$$

To obtain the contour integral estimate of $\Psi_{N-1}(x)$ we consider the function $f(z)$, where $z=x+i y$. By Cauchy's integral formula we can represent $f(x)$ by

$$
f(x)=\frac{1}{2 \pi i} \int_{C} \frac{f(z)}{z-x} d z,
$$

where $C$ is any contour on and within which $f(z)$ is regular. If the contour $C$ is so chosen that it contains the interval $-1 \leqq x \leqq 1$, then selecting the abscissas as the zeros of $T_{N+1}^{\prime}(x)$, the Lagrange interpolation polynomial for $f(x)$ can be written using [6, Section 3.6, p. 67] as

$$
\psi_{N-1}(x)=\frac{1}{2 \pi i} \int_{C} \frac{f(z)}{(z-x) T_{N+1}^{\prime}(z)}\left[T_{N+1}^{\prime}(z)-T_{N+1}^{\prime}(x)\right] d z,
$$

where the error

$$
\Psi_{N-1}(x)=\frac{T_{N+1}^{\prime}(x)}{2 \pi i} \int_{C} \frac{f(z)}{(z-x) T_{N+1}^{\prime}(z)} d z .
$$

2. Error in the Filippi Quadrature Method. In the Filippi quadrature formula we have from (3)

$$
\int_{-1}^{1} f(x) d x \approx \int_{-1}^{1} \psi_{N-1}(x) d x=2 \sum_{i=0}^{M} B_{2 j+1, N},
$$

where $M=(N-1) / 2$, if $N$ is odd and $M=(N-2) / 2$ for even $N$. Substituting for $B_{2 i+1, N}$, the expression in (4), (9) becomes

$$
\int_{-1}^{1} f(x) d x \approx \sum_{i=1}^{N} \lambda_{i} f\left(x_{i}\right)
$$

where

$$
\lambda_{i}=\frac{4}{N+1} \sum_{j=0}^{M} \frac{\left(1-x_{i}^{2}\right)}{(2 j+1)^{2}} T_{2 i+1}^{\prime}\left(x_{i}\right) .
$$

The error in the quadrature formula follows from (8) as

$$
E_{N-1}(\psi)=\int_{-1}^{1} \Psi_{N-1}(x) d x=\frac{1}{\pi i} \int_{C} \frac{f(z) L_{N}(z)}{T_{N+1}^{\prime}(z)} d z,
$$

where we have put

$$
L_{N}(z)=\frac{1}{2} \int_{-1}^{1} \frac{T_{N+1}^{\prime}(x)}{z-x} d x .
$$

Equation (13) defines $L_{N}(z)$ as a single-valued analytic function in the $z$-plane with 
the interval $[-1,1]$ for $x$ deleted. In the following discussion we shall work out an error estimate of the quadrature formula but before that we prove a lemma for $L_{N}(z)$ for odd $N$.

3. Lemma for $L_{N}(z)$. Let us now introduce the mapping

$$
z=\frac{1}{2}\left(\xi+\xi^{-1}\right), \quad \xi=\rho e^{i \theta}, \quad 0 \leqq \theta \leqq 2 \pi .
$$

This maps the exterior of the unit circle $|\xi|=1$ conformally onto the $z$-plane with the interval $-1 \leqq x \leqq 1$ deleted. The circle $|\xi|=\rho, \rho>1$, maps onto the ellipse $\varepsilon_{\rho}$ with foci at $z= \pm 1$ and semiaxes $\frac{1}{2}\left(\rho+\rho^{-1}\right)$ and $\frac{1}{2}\left(\rho-\rho^{-1}\right)$. The lemma stated below gives a simple representation of $L_{N}(z)$ on $\varepsilon_{\rho}$.

Lemma. For $z \in \mathcal{E}_{\rho}$

$$
\left|L_{N}(z)\right| \leqq(N+1)\left[\frac{\sigma_{V, N+3}}{\rho^{2}-1}+\frac{1}{\rho^{N+1}} \frac{4(N+1)}{2 N+3}\right] \text { for odd } N,
$$

where

$$
\sigma_{x, N+3}=2 \sum_{m=1}^{N+1} \frac{1}{2 m+1} .
$$

Proof. Following Davis [6, Lemma 12.4.6, p. 311] we set $x=\cos \theta$ and transform (13) to the $\xi$ plane. Then,

$$
L_{N}(z)=(N+1) \int_{0}^{\pi} \frac{\xi^{-1}}{1-2 \xi^{-1} \cos \theta+\xi^{-2}} \sin (N+1) \theta d \theta .
$$

Now

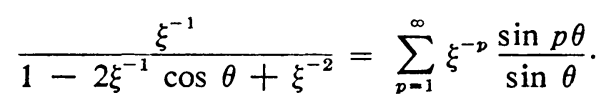

The series converges uniformly and absolutely for $0 \leqq \theta \leqq \pi$ and for all $|\xi| \geqq \rho>1$ Substituting (18) in (17),

$$
L_{N}(z)=(N+1) \sum_{p=1}^{\infty} \sigma_{N, p} \xi^{-p},
$$

where

$$
\sigma_{N, p}=\int_{0}^{\pi} \frac{\sin (N+1) \theta \sin p \theta}{\sin \theta} d \theta .
$$

From (20) we get

$$
\sigma_{N, p}=0 \text { if } N-p \text { is even, }
$$

$$
=2 \sum_{m=1}^{p} \frac{1}{N-p+2 m}, \quad \text { if } N-p \text { is odd. }
$$

It can now be easily verified that $\sum_{m=1}^{p} 1 /(N-p+2 m)$ assumes its maximum value when $N-p=-1$ for a fixed $N$. In that case $\sigma_{N, N+1}$ is the greatest coefficient in (19) and the next highest coefficient is $\sigma_{N, N+3}$, where

$$
\sigma_{N, N+1}=\sigma_{N, N+3}+\frac{4(N+1)}{2 N+3} .
$$


Now since $N$ is odd, by taking only even values of $p$ (say $2 r$ ), we have, from (19) and (22)

$$
L_{N}(z)<(N+1)\left[\sigma_{N, N+3} \sum_{r=1}^{\infty} \xi^{-2 r}+\frac{4(N+1)}{2 N+3} \xi^{-(N+1)}\right]
$$

and as $|\xi|=\rho>1$, the lemma is easily established.

4. Error Estimate for Analytic Functions. To obtain the error estimate for analytic functions in $[-1,1]$, a suitable choice of the contour in (12) is an ellipse as defined in (14). Now if $f \in A[-1,1]$, then for some $\rho>1, f$ can be continued analytically so as to be regular in the closed ellipse $\varepsilon_{\rho}$. Also on $\varepsilon_{\rho}$ both $T_{N+1}^{\prime}(z)$ and $L_{N}(z)$ have simple representations. That is, on $\varepsilon_{\rho}$

$$
T_{N+1}^{\prime}(z)=(N+1)\left\{\frac{\xi^{N+1}-\xi^{-(N+1)}}{\xi-\xi^{-1}}\right\}
$$

and

$$
\frac{|d z|}{\left|T_{N+1}^{\prime}(z)\right|} \leqq \frac{1}{2 \rho} \frac{\left(\rho+\rho^{-1}\right)^{2}}{\rho^{N+1}-\rho^{-(N+1)}} \frac{|d \xi|}{N+1} .
$$

Hence, applying the lemma for $L_{N}(z)$ ( $N$ odd), we have the following theorem:

THEOREM. Let $f \in A[-1,1]$ and be continuable analytically so as to be regular and single valued in the closed ellipse $\mathcal{E}_{\rho}$ with foci at $z= \pm 1$ and whose sum of the semiaxes is $\rho(\rho>1)$.

Then, from (12),

$$
\begin{aligned}
\left|E_{N-1}(\psi)\right| & =\frac{1}{\pi} \int_{\varepsilon \rho} \frac{|f(z)|\left|L_{N}(z)\right|}{\left|T_{N+1}^{\prime}(z)\right|}|d z| \\
& \leqq\left[\frac{\sigma_{N, N+3}}{\rho^{2}-1}+\frac{1}{\rho^{N+1}} \frac{4(N+1)}{2 N+3}\right] \frac{\left(\rho+\rho^{-1}\right)^{2}}{\rho^{N+1}-\rho^{-(N+1)}} M(\rho),
\end{aligned}
$$

where $M(\rho)=\max _{\varepsilon} \in \varepsilon_{\rho}|f(z)|$ on $\varepsilon_{\rho}$ (or equivalently on $|\xi|=\rho$ ).

Remarks. (1) The above estimate is poor for $\rho$ very nearly equal to 1 and is reasonably good for large $\rho$.

(2) It may be seen that for large $N$, the contribution from the second term in the bracket of (27) is negligible as compared to the first and hence,

$$
\left|E_{N-1}(\psi)\right| \leqq \frac{\sigma_{N, N+3}}{\rho^{2}-1} \frac{\left(\rho+\rho^{-1}\right)^{2}}{\rho^{N+1}-\rho^{-(N+1)}} M(\rho)
$$

holds approximately.

(3) To obtain the error estimate of the Clenshaw-Curtis quadrature scheme Chawla [4] uses interpolation points, $x_{i}=\cos \pi i / N, i=0(1) N(N$ even), whereas in the present estimate for the Filippi quadrature method, the corresponding points used are $x_{i}=\cos \pi i / N+1, i=1(1) N(N$ odd $)$.

Hence, if the same degree of the approximated polynomial is used for the quadrature problem in both cases, the value of $N$ in the present estimate must exceed by one the corresponding value in Chawla's estimate.

(4) It may be noticed that for large $N$, the expression $16 N^{2} /\left(4 N^{2}-1\right)$ (which 
TABLE 1

\begin{tabular}{cccc}
\hline$N$ & Present estimate & $N$ & Chawla's estimate \\
3 & 0.00166322 & 2 & 0.00423456 \\
5 & 0.00004028 & 4 & 0.00008230 \\
7 & 0.00000093 & 6 & 0.00000166 \\
9 & 0.000000020 & 8 & 0.000000034 \\
\hline
\end{tabular}

is slightly greater than 4) in Chawla's estimate [4, (24)] corresponds to $\sigma_{N, N+3}$ in the present estimate (28), the order for the remaining part being the same in both estimates. Also $\sigma_{N, N+3}$ is of the order $\log N$ for large $N$. Hence, if $\sigma_{N, N+3} \leqq 4$, the estimate (28) is better than Chawla's. And since the value of $N$ for which the above inequality holds is quite large, for practical purposes the estimate (28) can be conveniently used to obtain the error estimate in Filippi's quadrature.

5. Numerical Example. We now calculate the error estimate (27) for the Chebyshev quadrature for the function $f(x)=1 /(x+4)$ in $[-1,1]$ and compare the estimates with those obtained by Chawla [4]. We take as in [4, Section 5] $\rho=7$, $f(z)=1 /(z+4)$ on $\varepsilon_{\rho}$ and $M(\rho)=2.33333347$. The preceding table represents the estimations of error.

Acknowledgment. The author is grateful to Mr. P. K. Ghosh, Reader, Department of Applied Mathematics, Calcutta University for his kind guidance in the preparation of this paper and also to Prof. Dr. S. K. Chakravarty, Head of the Department of Applied Mathematics, Calcutta University for the facilities offered to him to work in the department. He also thanks the referee for suggestions for improving the paper.

\footnotetext{
Department of Applied Mathematics University College of Science 92, Acharya Prafulla Chandra Road Calcutta 9, India
}

1. S. FILIPPI, "Angenäherte Tschebyscheff-Approximation einer Stammfunktion-eine Modifikation des Verfahrens von Clenshaw und Curtis," Numer. Math., v. 6, 1964, pp. 320-328. MR 30 \#710.

2. C. W. Clenshaw \& A. R. CURtis, "A method for numerical integration on an automatic computer," Numer. Math., v. 2, 1960, pp. 197-205. MR 22 \#8659.

3. M. M. CHAWLA, "On the Chebyshev polynomials of the second kind," SIAM Rev., v. 9, 1967, pp. 729-733. MR $36 \# 5583$.

4. M. M. Chawla, "Error estimates for the Clenshaw-Curtis quadrature," Math. Comp., v. 22, 1968, pp. $651-656$. MR $37 \# 3753$.

5. M. M. ChAWLA \& M. K. JAIN, "Error estimates for Gauss quadrature formulas for analytic functions," Math. Comp., v. 22, 1968, pp. 82-90. MR $36 \# 6142$.

6. P. J. DAvIS, Interpolation and Approximation, Blaisdell, Waltham, Mass., 1963, pp. 67-68, 311-312. MR $28 \# 393$. 